\title{
Adaption of COBRA Method for The Investigation of Nutrition-Based DNA Methylation Mechanism in Escherichia coli
}

Taha Yasin KOÇ ${ }^{1}$, Medine GÜLLÜCE ${ }^{*}$, Burak ALAYLAR ${ }^{3}$, Mehmet KARADAYI $^{2}$

\begin{abstract}
Epigenetics are changes that occur in the DNA sequence due to environmental conditions. In last few decades epigenetics has been one of the most important advances in biology and related fields. It is very important in that it offers a new approach to all relevant disciplines, especially biology, to explain how DNA sequencing in an epigenetic organism is affected by environmental conditions and phenotype changes that may result from this interaction. Although there are many environmental factors affecting organisms, starvation is one of the most important factors. In our study, epigenetic changes in Escherichia coli due to carbon starvation in the $\operatorname{sprE}(\mathrm{rssB})$ gene region were investigated by COBRA (Combined Bisulfite Restriction Analysis) technique, which is one of the quantitative methylation analyzes. As a result of the current results, it has been shown that the methylation status of the 16th CpG islet located in the sprE gene region does not play a role in the regulation of fasting metabolism in $E$. coli due to the restriction of the carbon level.
\end{abstract}

Keywords: Capillary gel electrophresis, COBRA, DNA methylation, Escherichia coli, sprE gene

${ }^{1}$ Taha Yasin KOÇ (Orcid ID: 0000-0002-7786-5462), Atatürk University, Institute of Natural and Applied Sciences, Erzurum, Turkey

${ }^{2}$ Medine GÜLLÜCE (Orcid ID: 0000-0002-5957-8259), Atatürk University, Faculty of Science, Department of Biology, Erzurum, Turkey

${ }^{3}$ Burak ALAYLAR (Orcid ID: 0000-0001-6737-3440), Agri Ibrahim Cecen University, Faculty of Arts and Sciences, Department of Molecular Biology and Genetics, Agri, Turkey

${ }^{2}$ Mehmet KARADAYI (Orcid ID: 0000-0002-2473-0409), Atatürk University, Faculty of Science, Department of Biology, Erzurum, Turkey

*Corresponding Author: Medine GÜLLÜCE, e-mail: gullucem@atauni.edu.tr 


\section{INTRODUCTION}

The term epigenetics was first introduced to explain how cell lines were shaped during the development of multicellular eukaryotes in 1942 by Conrad Waddington, (Handy et al., 2011; Tollefsbol, 2011; Yaykaşl1 et al., 2012; Casadesûs and Low, 2013; Gulluce et al., 2014; Jurkowski et al., 2015). It is widely known that epigenetic control of gene expression plays an important role in the processes of cell differentiation in vertebrates (Takahashi, 2013). At this point, most textbooks and study books on epigenetic gene regulation only concern eukaryotes. One reason for this is the great success of eukaryotic epigenetics and its effects on human diseases (Casadesûs and Low, 2013).

Although there are many different types of classification, epigenetic mechanisms that regulate gene expression fall into two sub-categories, mainly indirect and direct role mechanisms. Among these, the mechanisms that directly take part; Acetylation, methylation, phosphorylation, S-nitrosylation, SUMOylation, ADP-ribosylation, proline isomerization, deimination and non-covalent histone modifications (Gulluce et al., 2014; Alaylar et al., 2019). All these epigenetic modifications are known to be altered by external and internal environmental factors and affect gene expression. Thus, epigenetics can provide a new research framework for environment-related etiologic factors, as well as embryonic development and aging are known to be affected by many environmental factors (Choi and Friso, 2010).

Nutrition is defined as one of the most important environmental factors known. Therefore epigenetics is extremely important as it can alter the gene expression and epigenetic events of nutrients and bioactive food components at transcriptional levels. Folate, vitamin B-12, methionine, choline and betaine may affect DNA methylation and histone methylation through alteration of one carbon metabolism (Choi and Friso, 2010; Takahashi, 2013).

It is known that DNA methylation is one of the most important nutrient-related epigenetic mechanisms. This phenomenon is a vital mechanism in normal embryonic development. The DNA methylation event, which changes a cytosine base in $\mathrm{CpG}$ dinucleotide residues with methyl groups, is catalyzed by Dnmt (DNA methyl-transferase enzymes) and modulates gene expression motifs by altering chromatin structures (Choi et al., 2009; Lillycrop and Burdge, 2015; Warzak et al., 2015).

Natural microbial environments often require adaptation to various stresses, such as food hunger, which can occur quickly. Escherichia coli responds to fasting stress by entering a state where it is metabolically less active, known as the fixed phase. As one of the proteins with the highest degree of expression regulation in E.coli, RpoS draws attention. After binding of the $s p r E$ protein to RpoS, RpoS accumulation in cells is thought to be affected and regulates the transcription of target genes (Carabetta et al. 2009).

Many techniques have been developed to show methylation events, which are largely responsible for the emergence of epigenetic arrangements in living things, and especially methods based on bisulfite reaction that allows rapid diagnosis of methylated cytosine (5-MeC) in DNA. Methylation-specific PCR (MSP), bisulfite sequencing and COBRA (combined bisulfite restriction analysis) methods are among the most well-known of these applications (Kosova et al., 2011). Among all these methods aimed at measuring the methylation status of DNA, applications involving the combination of PCR and Restriction enzymes have given a new perspective and momentum to existing epigenetic studies by providing quantitative results (Alaylar, 2014).

In this study was designed to quantitatively investigate the methylation motifs that may occur in the regulation of the $\operatorname{sprE}$ ( $r s s B$ ) gene region activated by nutritional starvation stress in Escherichia coliby COBRA method. Also in the current study, it is aimed to contribute to filling the theoretical gap related to epigenetic mechanisms existing in prokaryotic organisms. 


\section{MATERIALS AND METHODS}

\section{Chemicals}

Sodium chloride $(\mathrm{NaCl})$ was provided from Merck (Hohenbrunn, Germany). Chloroform-isoamyl alcohol were provided Fluka (Steinheim, Germany), phenol-chloroform-isoamyl alcohol 25:24:1 were provided Amresco. All other compounds such as; isopropanol, glycerol, ethylenediaminetetraacetic acid (EDTA), Tris-HCl, Direct LoadTM wide-range DNA marker, CTAB, SDS, RNAse, DMSO, Trizma base, Ethidium bromide and ethanol were purchased from Sigma-Aldrich (St. Louis, USA). The restriction enzymes for the COBRA analysis, TaqI and HinfI were obtained Thermo-Scientific (Waltham, MA USA). Wizard genomic DNA purification kit and MethyledgeTM bisulfite conversion system were purchased from Promega (Madison, USA). Agarose was provided by Lonza. Expand high fidelity PCR system was acquired from Roche (Mannheim, Germany). Nutrient Broth and Nutrient Agar, Potato Dextrose Agar, bacteriological peptone and agarose acquired from Oxoid (Hampshire, England), LAB M (Lancashire, England) and Lonza (Rockland, USA) respectively.

\section{Escherichia coli Strain}

The bacterial strain Escherichia coli WP2uvrA (ATTC® Number: 49979) used in this study was commercially available from LGC Standards (Middlesex, U.K.).

\section{Medium}

Carbon Source Restricted Test Media is used to expose the test microorganism to carbon deficit. In preparation of this medium, $980 \mathrm{ml}$ of distilled water is sterilized by autoclaving for 30 minutes at $121^{\circ} \mathrm{C}$. After this process, the previously sterilized Vogel-Bonner (VB salts) medium E $(50 \times)$ solution is added to the sterile water medium whose temperature is lowered to $45-65^{\circ} \mathrm{C}$, and it is mixed well by gentle agitation in the sterile cabinet. This medium should be stored in sterile environments at room temperature (Mortelmans and Zeiger, 2000; Karadayı, 2010).

\section{Preparation of Test Samples}

Stock cultures were thawed at room temperature before working cultures were prepared. Then, $200 \mu \mathrm{l}$ of stock culture was added to NB liquid medium, each having a volume of $40 \mathrm{ml}$. Prepared working cultures were allowed to incubate at $37^{\circ} \mathrm{C}$ at $110 \mathrm{rpm}$ for 48 hours. After this time, one of the growing group cultures was transferred to a new NB medium and the other group was transferred to a carbon-restricted medium and these new cultures were left to the second 24-hour incubation period. DNA isolation, sodium bisulfite application, High Fidelity PCR, restriction enzyme cutting and imaging applications were performed with these cultures.

\section{Genomic DNA Isolation}

Genomic DNA was isolated from Escherichia coli. DNA isolation protocol was explained as follows.

Cultures were grown in NA media, and then two loops of bacteria are placed in eppendorf and shaken well after vortexing $1000 \mu \mathrm{l}$ of STE buffer is added. The samples are centrifuged at $10000 \mathrm{rpm}$ for 10 minutes and the supernatant is discarded. $1000 \mu 1$ of STE (Sodium Chloride-Tris-EDTA) buffer is then added again and centrifuged at $10000 \mathrm{rpm}$ for 10 minutes at $4{ }^{\circ} \mathrm{C}$. After the centrifugation process is completed, the supernatant is discarded and $500 \mu \mathrm{l}$ of STE buffer is added to the pellet and mixed in every 15 minutes for a total of 30 minutes in the water bath previously set to $75{ }^{\circ} \mathrm{C}$. At the end of the period, $10 \%$ SDS is added to eppendorf and mixed. After this, $8 \mu \mathrm{l}$ of Proteinase $\mathrm{K}$ is added and shaken again. After rinsing, it is suspended for 1 hour in a water bath set to $40{ }^{\circ} \mathrm{C}$. Add $1 / 5$ of the total volume, $5 \mathrm{M} \mathrm{NaCl}$ to the samples taken from the water bath. $10 \%$ by volume of CTAB 
(Hexadecyltrimethylammonium bromide) is added to the resulting new volume sample and shaken by finger. The sample is stored in a water bath set at $65{ }^{\circ} \mathrm{C}$ for 10 minutes. Then, the total volume of phenolchloroform-isoamyl alcohol (25: 24: 1) is added to the sample and rinsed with a rotator for 15 minutes. After shaking, centrifugation is performed at $16000 \mathrm{rpm}$ for 3 minutes at $3{ }^{\circ} \mathrm{C}$. After centrifugation, the supernatant is transferred to a sterile eppendorf and after $10 \% \mathrm{CTAB}$ of volume is added, it is allowed to stand for 10 minutes in a water bath set at $65^{\circ} \mathrm{C}$. An equal volume of chloroform-isoamyl alcohol (24:1) is added to the sample removed from the water bath. After the addition, the sample is shaken with the rotator for 15 minutes and then centrifuged at $16000 \mathrm{rpm}$ for 10 minutes. After centrifugation, the supernatant is transferred to the sterile eppendorf. $60 \%$ of the sample transferred to the sterile eppendorf is transferred to isopropanol stored at $-20^{\circ} \mathrm{C}$ and incubated overnight at $-20^{\circ} \mathrm{C}$ in the freezer. The sample taken from the freezer is centrifuged at $15000 \mathrm{rpm}$ at $3{ }^{\circ} \mathrm{C}$ for 10 minutes. $70 \%$ ethanol is added to the sample that has finished centrifugation and then centrifuged at $15000 \mathrm{rpm}$ for 3 minutes at $3{ }^{\circ} \mathrm{C}$. After centrifugation, the supernatant is decanted and allowed to dry at room temperature for one hour. After removal of alcohol, $70 \mu \mathrm{l}$ of TE (Tris-EDTA) buffer is added and $3 \mu 1$ of RNAase is added and allowed to dry for one hour at room temperature. Store at $-20{ }^{\circ} \mathrm{C}$. The results of DNA isolation were shown in Figure 1 (Bozoğlu, 2014).

\section{Bisulfite Conversion Reaction Step}

The kit was supplied from the Promega company for the bisulfite conversion reaction and the application was carried out according to the kit protocol provided by the company.

\section{Sodium Bisulfite Treatment}

Many bisulfite conversion kits have been advanced for bisulfite treatment because it is hard to succeed and optimal balance between complete conversion and minimal degradation of DNA. In this present study, DNA treatment with sodium bisulfite was performed using the Methyledge ${ }^{\mathrm{TM}}$ bisulfite conversion kit (Promega, San Luis Obispo-USA) according to the manufacturer's protocol. Also specific primer sets were designed after the bisulfite treatment for $s p r E$ gene region. Detailed information of primer set for related genes were shown in Table 1.

Table 1. Primer sets for $\operatorname{sprE}$ gene region

\begin{tabular}{ll}
\hline T2 Primer & \\
\hline Forward & TGATATCGCGATGCCACGAA \\
Reverse & CAATAACGCGCCCAACTCTG \\
Product length & 565 base pair \\
\hline
\end{tabular}

\section{T7 Primer}

\begin{tabular}{ll}
\hline Forward & GTAACAGAGGCGACCAGACC \\
Reverse & GGCAATGGGAAATCACCTGC \\
Product length & 294 base pair \\
\hline
\end{tabular}

\section{PCR Protocol}

Samples were prepared for as a volume of 30 PCR reactions for each reaction. Each PCR reaction was contained 16.1 $\mu$ l distilled water, $3 \mu 1$ 10xPCR buffer, $1.2 \mu 1 \mathrm{DMSO}, 1.8 \mu 1 \mathrm{MgCI}_{2}$ solution (25mM), $3 \mu 1$ Forward primer, $3 \mu 1$ Reverse primer, $0.6 \mu 1$ dNTPs (deoxynucleotide triphosphates: dATP, dGTP, dCTP, dTTP), $0.3 \mu 1$ Taq DNA polymerase enzyme and $1 \mu$ l bisulfite-treated DNA.

PCR conditions were as follows: $95^{\circ} \mathrm{C}$ for 2 minutes, 36 cycles of denaturing at $94{ }^{\circ} \mathrm{C}$ for 1 minute, 1 minute annealing at $58{ }^{\circ} \mathrm{C}$ (This step should prepare for each primer Tm temperature), 2 minutes and 5 minutes extension steps at $72^{\circ} \mathrm{C}$ (Warzak et al., 2015). 


\section{Digestion of PCR Products with HinfI Restriction Enzyme}

Digestion of PCR products was carried out by restriction enzyme HinfI enzyme following the following steps;

- $10 \mu \mathrm{l}$ of PCR product, $18 \mu \mathrm{l}$ of distilled water, $2 \mu \mathrm{l}$ of 10X buffer R (compatible buffer) HinfI and 2 $\mu \mathrm{l}$ of HinfI enzyme are added. Mix vigorously and allow to incubate for 1-16 hours.

- The restriction enzyme is kept at $65^{\circ} \mathrm{C}$ for 20 minutes in order to stop the reaction.

- The restriction products are stored at $+4{ }^{\circ} \mathrm{C}$.

After this application the digested PCR products were evaluated with the QIAxcel Advanced analysis system.

\section{Restriction Enzymatic Digestion of PCR Products}

RestrictionMapper program (http://www.restrictionmapper.org) is used to determine the restriction sites in the products to be obtained after the amplification of $s p r E$ gene region with the designed specific primer sets. Restriction sites for PCR products of $s p r E$ gene information were given in Table 2. Enzymatic digestion of PCR product was performed with HinfI. These restriction enzymes were provided from Thermo-Scientific (Waltham, MA USA).

Table 2. Restriction sites of the PCR product of the $\operatorname{sprE}$ gene region

\begin{tabular}{ccc}
\hline Restriction Enzyme Name & Sequence & Cut Position \\
\hline HinfI & GANTC & 339,974 \\
\hline
\end{tabular}

After this step the digested PCR products were analyzed by QIAxcel advanced analysis system.

\section{Selecting the Gene Region to Be Used in the Study}

In this study, the basic gene region of $\operatorname{sprE}$ ( $r s s B$ ) related to nutrient starvation was selected for the applications. The known sequence information for this gene region is shown below.

\section{(https://www.ncbi.nlm.nih.gov/nuccore/NC_000913.3? report=fasta\&from=1290242\&to=1291255)}

ATGACGCAGCCATTGGTCGGAAAACAGATTCTCATTGTTGAAGATGAGCAGGTATTTCGCTCGCTTCTGGATT CATGGTTTTCCTCATTGGGAGCGACAACGGTACTGGCGGCTGATGGGGTGGATGCCCTTGAGTTGCTGGGAG GTTTCACTCCAGACCTGATGATATGTGATATCGCGATGCCACGAATGAACGGGCTTAAACTGCTGGAGCATA TACGTAACAGAGGCGACCAGACCCCAGTTCTGGTGATATCTGCCACTGAAAATATGGCAGATATTGCCAAAG CGTTACGTCTGGGCGTTGAAGATGTTTTGCTGAAACCAGTTAAAGATCTGAATCGCTTGCGCGAGATGGTTTT TGCCTGTCTCTATCCCAGCATGTTTAATTCGCGCGTTGAGGAAGAGGAAAGGCTTTTTCGCGACTGGGATGCA ATGGTTGATAACCCTGCCGCAGCGGCGAAATTATTACAGGAACTACAACCGCCGGTTCAGCAGGTGATTTCC CATTGCCGGGTTAATTATCGTCAATTGGTTGCCGCGGACAAACCCGGCCTGGTGCTTGATATTGCCGCACTTT CGGAAAACGATCTGGCATTTTATTGCCTTGATGTCACCCGAGCTGGACATAATGGCGTACTTGCTGCCTTGTT ATTACGCGCATTGTTTAACGGATTATTACAGGAACAGCTTGCACACCAAAATCAACGGTTGCCAGAGTTGGG CGCGTTATTGAAGCAGGTAAACCATTTACTTCGTCAGGCCAATCTGCCGGGGCAGTTTCCGCTATTAGTTGGC TATTATCATCGCGAACTGAAAAATCTCATTCTGGTTTCTGCGGGTCTGAATGCGACGTTAAATACCGGCGAAC ACCAGGTGCAAATCAGTAATGGTGTTCCGTTAGGCACTTTAGGTAACGCTTATTTGAATCAATTGAGCCAGCG ATGCGATGCCTGGCAATGCCAAATATGGGGAACCGGTGGTCGACTGCGCTTGATGTTGTCTGCAGAATGA

The results of PCR performed to confirm the presence of the sprE gene region in Escherichia coli WP2uvrA bacterial strain, our experimental microorganism, are shown in Figure 1. 


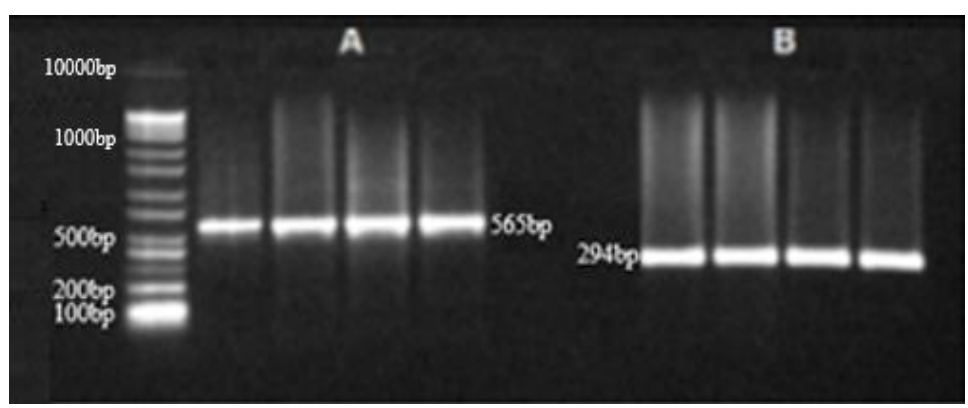

Figure 1. Demonstration of the presence of the $\operatorname{sprE}$ gene region in the Escherichia coli WP2uvrA bacterial strain.

* A: T2 primer, $\mathrm{B}: \mathrm{T} 7$ primer.

Determination of Methylation Status Change That may Ocur in Case of Carbon Starvation in Selected CpG Islets in the sprE ( $r s s B$ ) Gene Region by COBRA Method

\section{Bisulfite-treated sprE (rssB) Gene Region TAHA2 Primer}

Sequence information of the $\operatorname{sprE}$ ( $r s s B$ ) gene region after bisulfite application and $\mathrm{CpG}$ islands were given Figure 2.

Table 3. TAHA2 Primers

\begin{tabular}{ll}
\hline Primers & \\
\hline Forward & GAGGYGATTAGATTTTAGTTTTGGTGATATTTG \\
Reverse & TACRACAATATCAAACACCAAACC \\
\hline
\end{tabular}

ATGACGTAGTTATTGGTCGGAAAATAGATTTTTATTGTTGAAGATGAGTAGGTATTTCG TTCGTTTTTGGATTTATGGTTTTTTTTATTGGGAGCGATAACGGTATTGGCGGTTGATG GGGTGGATGTTTTTGAGTTGTTGGGAGGTTTTATTTTAGATTTGATGATATGTGATATC GCGATGTTACGAATGAACGGGTTTAAATTGTTGGAGTATATACGTAATAGAGGCGATTA GATTTTAGTTTTGGTGATATTTGTTATTGAAAATATGGTAGATATTGTTAAAGCGTTAC GTTTGGGCGTTGAAGATGTTTTGTTGAAATTAGTTAAAGATTTGAATCGTTTGCGCGAG ATGGTTTTTGTTTGTTTTTATTTTAGTATGTTTAATTCGCGCGTTGAGGAAGAGGAAAG GTTTTTTCGCGATTGGGATGTAATGGTTGATAATTTTGTCGTAGCGGCGAAATTATTAT AGGAATTATAATCGTCGGTTTAGTAGGTGATTTTTTATTGTCGGGTTAATTATCGTTAA TTGGTTGTCGCGGATAAATTCGGTTTGGTGTTTGATATTGTCGTATTTTCGGAAAACGA TTTGGTATTTTATTGTTTTGATGTTATTCGAGTTGGATATAATGGCGTATTTGTTGTTT TGTTATTACGCGTATTGTTTAACGGATTATTATAGGAATAGTTTGTATATTAAAATTAA CGGTTGTTAGAGTTGGGCGCGTTATTGAAGTAGGTAAATTATTTATTTCGTTAGGTTAA TTTGTCGGGGTAGTTTTCGTTATTAGTTGGTTATTATTATCGCGAATTGAAAAATTTTA TTTTGGTTTTTGCGGGTTTGAATGCGACGTTAAATATCGGCGAATATTAGGTGTAAATT AGTAATGGTGTTTCGTTAGGTATTTTAGGTAACGTTTATTTGAATTAATTGAGTTAGCG ATGCGATGTTTGGTAATGTTAAATATGGGGAATCGGTGGTCGATTGCGTTTGATGT

Figure 2. Sequence information of the $\operatorname{sprE}(\mathrm{rssB})$ gene region after bisulfite application and $\mathrm{CpG}$ islands.

\section{Bisulfite Treatment and PCR Reaction}

Specific products were obtained by PCR application with Taha-2 primer specially designed for bisulfite treatment after DNA isolation studies of E.coli samples developed on carbon restricted medium and Nutrient Broth (NB) media. The agarose gel (\%1) image of these findings is shown in Figure 3. 


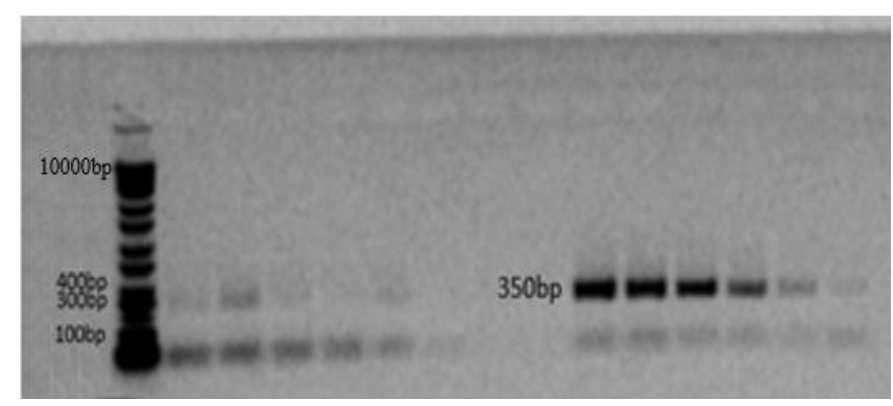

Figure 4. Results of PCR with Taha-2 Primer

\section{RESULTS AND DISCUSSION}

\section{sprE (rssB) PCR Products Restriction Application}

Restriction application studies were performed with HinfI enzyme for $\operatorname{sprE}$ ( $r s s B$ ) PCR products. QIAxcel Advanced analysis results of this study are shown in Figure 5, Figure 5.1, Figure 5.2, Figure 5.3, Figure 5.4 and Figure 5.5.

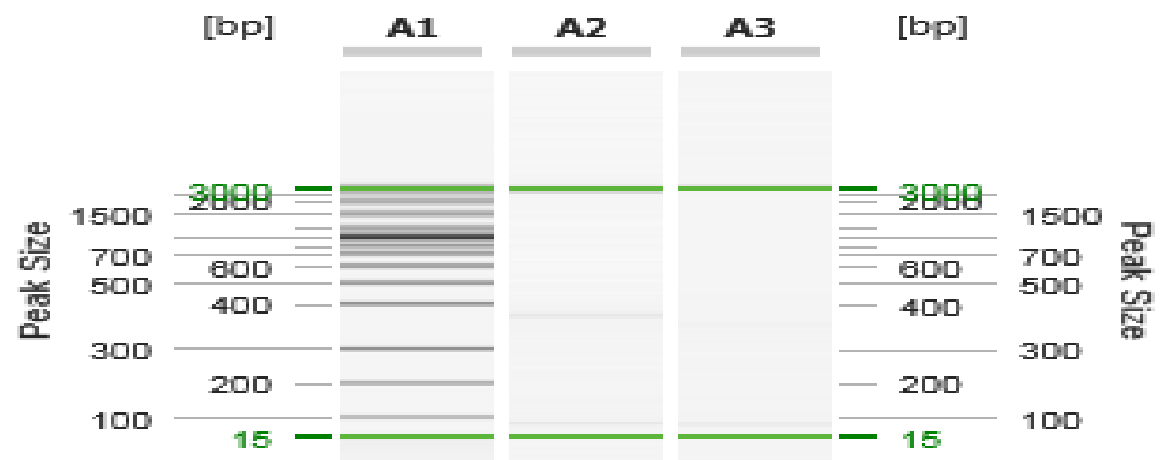

Figure 5. sprE gene region of organism developed on NB medium COBRA gel image analysis result

* A1: Marker, A2: PCR product after bisulfite application, A3: The product after restriction application.

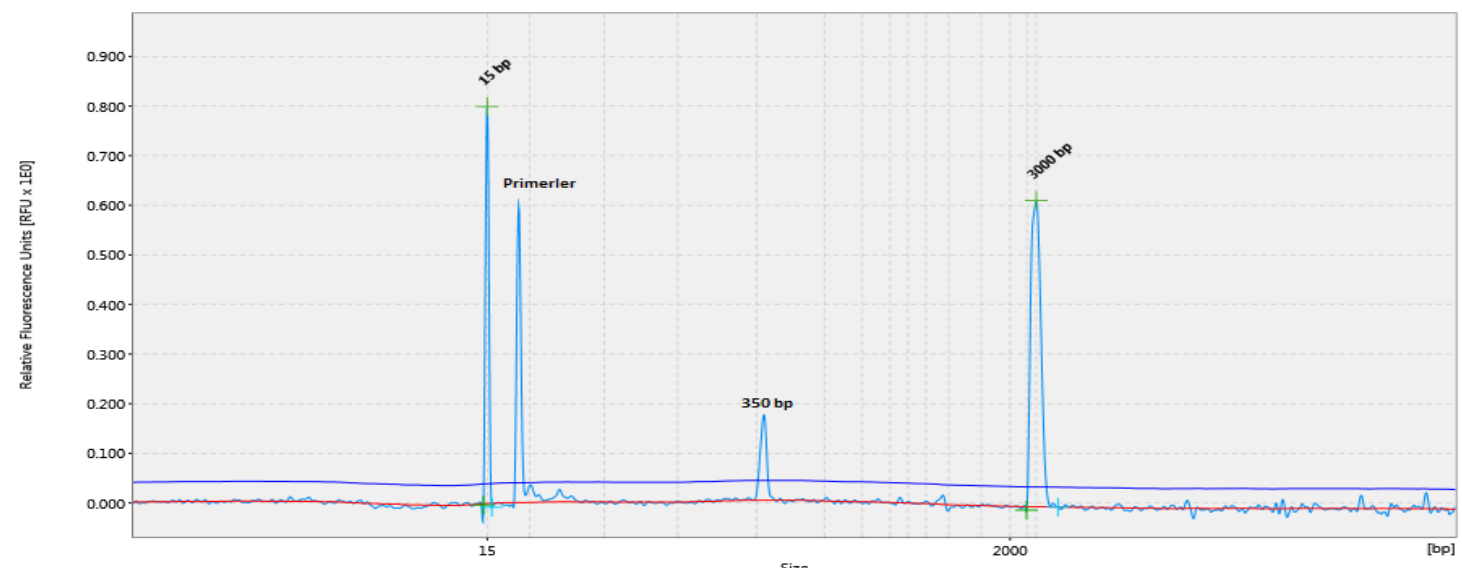

Figure 5.1. PCR product electrophoresis after bisulfite application for the $\operatorname{sprE}$ gene region of the organism developed on NB medium. 


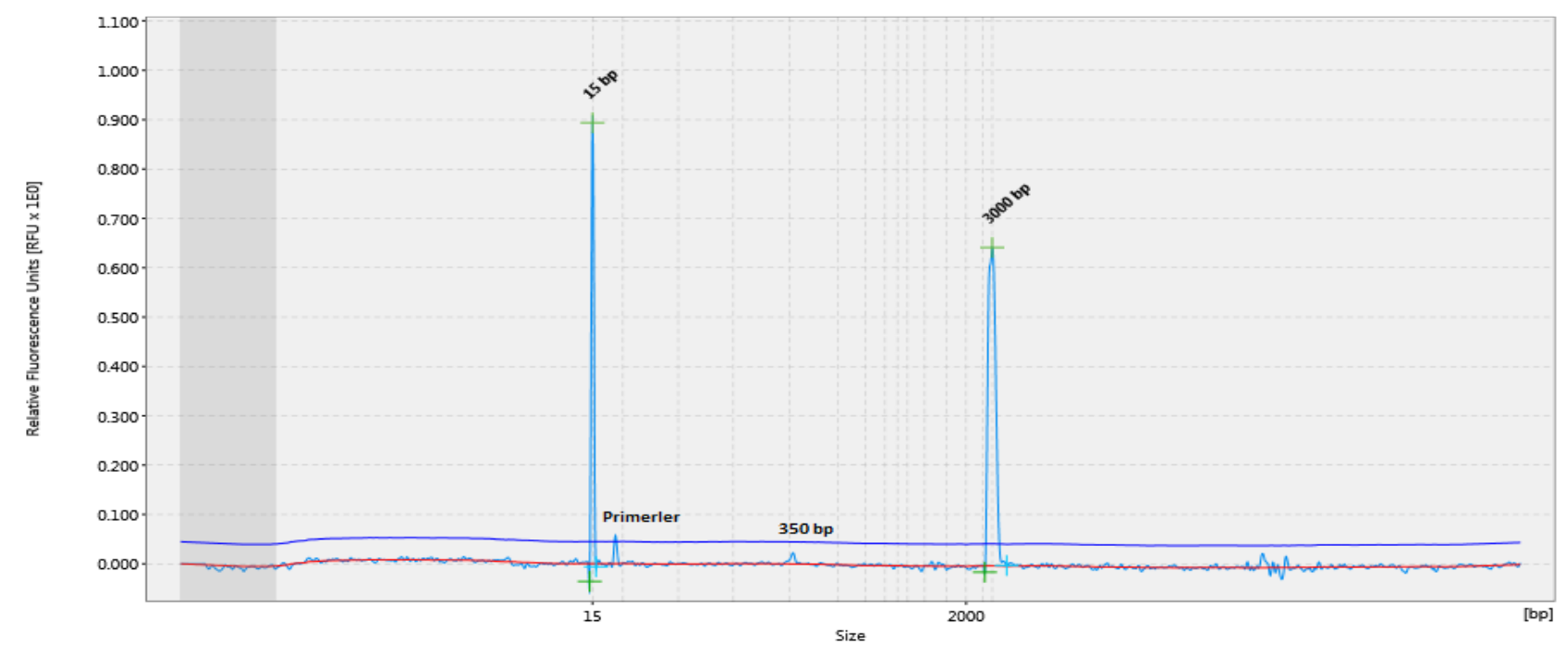

Figure 5.2. Electrophoresis of the product after restriction application for the $s p r E$ gene region of the organism developed on NB medium.

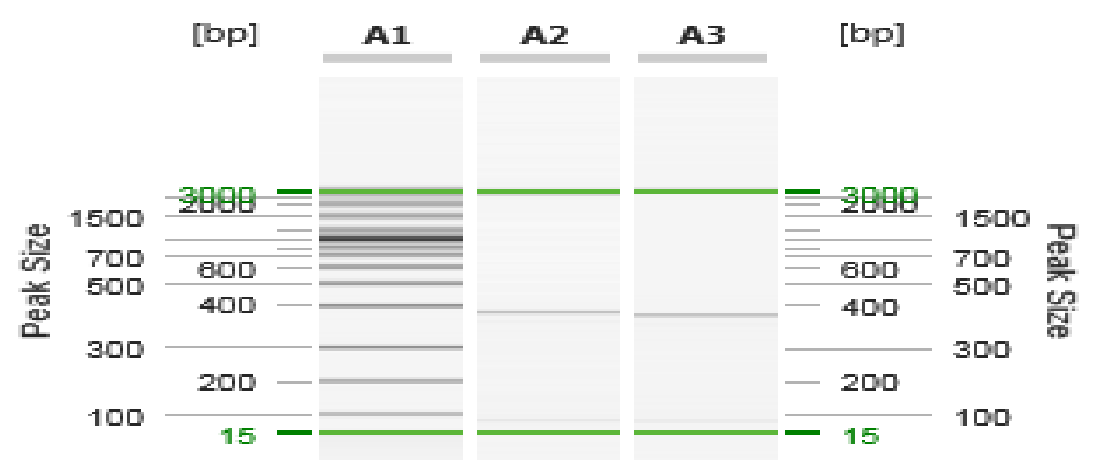

Figure 5.3. COBRA gel image analysis of the $s p r E$ gene region of the organism developed on carbon-restricted medium

*A1: Marker, A2: PCR product after bisulfite application, A3: Product after restriction application

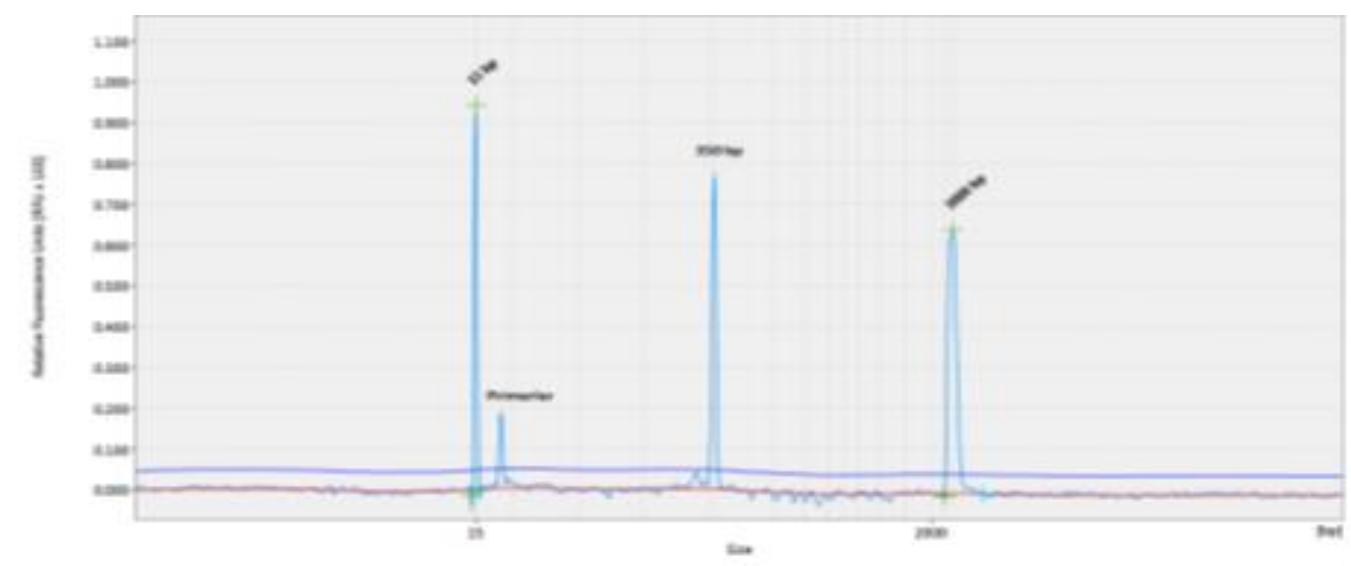

Figure 5.4. PCR product electropherogram after bisulfite application for the $\operatorname{sprE}$ gene region of the organism developed on carbon-restricted medium 


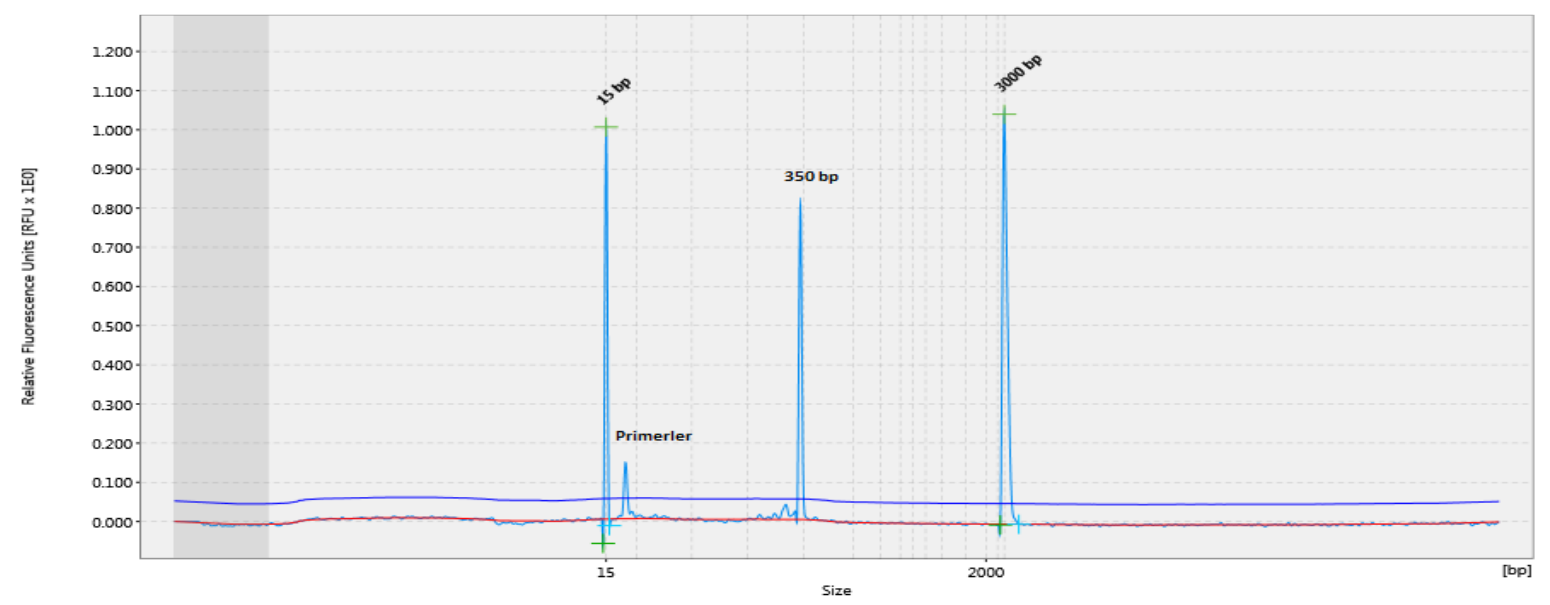

Figure 5.5. Electrophoresis of the product after restriction application for the $\operatorname{sprE}$ gene region of the organism developed on carbon-restricted media

When the research findings are examined, this region is not methylated in both of the bacterial groups grown in the carbon-rich environment and in the poor environment, so that the DNA fragment is not cut due to the $\mathrm{C} \rightarrow \mathrm{T}$ transformation occurring in the recognition region of the enzyme as a result of the cutting application performed with the HinfI restriction enzyme and finally, it was concluded that the methyl profile of the $\mathrm{CpG}$ islet had no effect on the regulation of the gene in the case of carbon starvation due to the fact that the $\operatorname{sprE}(r s s B$ ) gene region of the E.coli WP2uvrA bacterial strain did not change due to development in the carbon-rich and poor environments.

\section{CONCLUSION}

In this study, it was aimed to quantitatively investigate methylation motifs that may occur in regulation of $\operatorname{sprE}(r s s)$ gene region which is activated as a result of nutritional starvation in model prokaryotic microorganism Escherichia coli by COBRA method.

For this purpose, the restriction enzyme was examined in silico applications in HinfI restriction enzyme experiments depending on the formation of the COBRA analysis of the carbon-rich 16.CpG islets of the $\operatorname{sprE}(r s s)$ gene region of the E.coli WP2uvrA bacterial strain and the development of the methylation status.

In addition, the use of capillary gel electrophoresis technique for such sensitive applications appears to be the most efficient method of presenting the separation of both small and large molecules. Accordingly, a wide range of analytical diversity, high mass sensitivity, high efficiency separation, the ability to work with very low volumes, short analysis times, minimal use of consumables and basic device setup, such as the convenience of a number of features that highlight the use of capillary gel electrophoresis technique highlights (Karakaş ve Certel, 2006). When our findings are taken into consideration, it is clearly shown that the experimental results obtained after bisulfite applications which cause high product loss can be analyzed more precisely by using capillary electrophoresis systems.

As a result, the data obtained from our present study indicate that COBRA technique can be used effectively in prokaryotic systems in the investigation of DNA methylation profile changes due to epigenetic events, similar to the applications of many eukaryotic systems in the literature (Xiong and Laird, 1997; Goedecke et al., 2009). In this respect, our present study differs from other applications performed with eukaryotic systems and shows originality. However, it has been shown that the sensitivity of the technique can be increased by adapting the capillary electrophoresis system to the common COBRA method which is known with our present study. 


\section{REFERENCES}

Alaylar B, 2014. Determination of Epigenetic Changes Depending on Presence of Sucrose and Glucose in Related Gene Regions of Saccharomyces cerevisiae. Atatürk University Graduate School of Natural and Applied Sciences, Master Thesis (Printed).

Alaylar B, Gulluce M, Karadayı M, 2019. Development and Evaluation of Epigenetic Regulation of Sucrose Metabolism in Saccharomyces cerevisiae by Using COBRA Technique and Selected CpG Islands in HXT10 and SUC2 genes. Eastern Anatolian Journal of Science, 5, 50-55.

Bozoğlu C, 2014. Purification of Laccase Enzyme of Thermophilic Brevibacillus sp. Isolated from Agri Diyadin Hot Spring and Determination of Potential Industrial Usage of the Enzyme. Atatürk University Graduate School of Natural and Applied Sciences, Master Thesis (Printed).

Carabetta, V.J., Mohanty, B.K., Kushner, S.R., Silhavy, T.J., 2009. The Response Regulator sprE (rssB) Modulates Polyadenylation and mRNA Stability in Escherichia coli. Journal of Bacteriology, 22, 6812 -6821 .

Casadesûs J, Low DA, 2013. Programmed Heterogeneity: Epigenetic Mechanisms in Bacteria. The Journal of Biological Chemistry, 288, 13929-13935.

Choi SW, Friso S, 2010. Epigenetics: A New Bridge between Nutrition and Health. Advances in NutritionAn International Review Journal, 1, 8-16.

Choi SW, Corrocher R, Friso S, 2009. Nutrients and DNA Methylation. Nutrients and Epigenetics, Ed: Choi, S.W., Friso, S. CRC Press Taylor \& Francis Group, Boca Raton, 105-125.

Goedecke S, Schlosser S, Mühlisch J, Hempel G, Frühwald MC, Wünsch B, 2009. Determination of DNA methylation by COBRA: A comparative study of CGE with LIF detection and conventional gel electrophoresis. Electrophoresis, 30, 3063 - 3070.

Gulluce M, Alaylar B, Koc TY, Karadayı M, 2014. Epigenetics: An Innovative Approach for Biotechnology and Food Science. International Journal of Bioscience, Biochemistry and Bioinformatics, 4 (3), 195 199.

Handy DE, Castro R, Loscalzo J, 2011. Epigenetic Modifications Basic Mechanisms and Role in Cardiovascular Disease. Circulation Journal of American Heart Association, 123, 2145-2156.

Jurkowski TP, Ravichandran M, Stepper P, 2015. Synthetic Epigenetics-Towards Intelligent Control of Epigenetic States and Cell Identity. Clinical Epigenetics, 7 (18), 1 - 11.

Karadayı M, 2010. Determination of Mutagenic and Antimutagenic Properties of Some Active Compounds from Origanum vulgare L. ssp. vulgare Using Ames/Salmonella and E. coli Wp2 Test Systems. Atatürk University Graduate School of Natural and Applied Sciences, Master Thesis (Printed).

Karakaş B, Certel M, 2006. Kapiler Elektroforez Tekniği ve Gıda Analizlerinde Kullanım Olanakları - Tahıl Proteinlerinin Analizine Getirdiği Açılımlar. Türkiye 9. Gıda Kongresi, Bolu.

Kosova B, Özel R, Aktan Ç, 2011. Has DNA Methylation any Impact in Prostate Cancer Diagnosis? Bulletin of Urooncology, 2, 33 - 40.

Lillycrop KA, Burdge GC, 2015. Maternal Diet as a Modifier of Offspring Epigenetics. Journal of Developmental Origins of Health and Disease, 6 (2), 88 -95.

Mortelmans K, Zeiger E, 2000. The Ames Salmonella/Microsome Mutagenicity Assay. Mutation Research, 455, $29-60$.

Szarc vel Szic K, Declerck K, Vidakovic M, Berghe WV, 2015. From Inflammaging to Healthy Aging by Dietary Lifestyle Choices: Is Epigenetics the Key to Personalized Nutrition? Clinical Epigenetics, 7 (33), $1-18$.

Takahashi K, 2013. Influence of Bacteria on Epigenetic Gene Control. Cellular and Molecular Life Sciences, 71, 1045- 1054. 
Tollefsbol TO, 2011. Epigenetics: The New Science of Genetics, Chapter 1. Handbook of Epigenetics-The New Molecular and Medical Genetics, Ed: Trygve Tollefsbol. University of Alabama at Birmingham, Birmingham, 1 - 6.

Tollefsbol, TO, 2004. Methods of Epigenetic Analysis, Cahapter 1. Epigenetics Protocols, Ed: Trygve Tollefsbol. Humana Press, New Jersey, $1-9$.

Warzak DA, Johnson SA, Ellersieck MR, Roberts RM, Zhang X, Ho Shuk-Mei, Rosenfeld CS, 2015. Effects of Post-Weaning Diet on Metabolic Parameters and DNA Methylation Status of the Cryptic Promoter in the Avy Allele of Viable Yellow Mice. Journal of Nutritional Biochemistry, 26, 667 - 674.

Xiong Z, Laird PW, 1997. COBRA: A Sensitive and Quantitative DNA Methylation Assay. Nucleic Acids Research, 25 (12), 2532 - 2534.

Yaykaşı KO, Hatipoğlu ÖF, Kaya E, Yaykaşı E, 2012. Epigenetics Mechanisms and Cancer. Düzce Medical Journal, 14 (3), 58 - 68. 